\title{
A Unified Dual-Stage Actuator Control Scheme for Track Seeking and Following in Hard Disk Drives
}

\author{
Jinchuan Zheng and Minyue Fu
}

\begin{abstract}
This paper presents a unified approach to track seeking and following control of a hard disk drive (HDD) dual-stage actuator (DSA) system. Based on doubly coprime factorization (DCF) method, the DSA controller and the closedloop dynamics are expressed explicitly in terms of two design parameters. This greatly simplifies the optimization of design parameters in meeting desired specifications. We then address how to use the design parameters to deal with specific problems in the DSA, i.e., control allocation for disturbance rejection and trajectory planning for track seeking. Simulated results are also presented to verify the effectiveness of the proposed DSA controller. Compared to previous works, the proposed approach can fulfill track seeking and following tasks by a single controller without switching. Moreover, the unified controller can achieve desirable performances for both tasks which are equivalent to that by two separate conventional controllers.
\end{abstract}

\section{INTRODUCTION}

Hard disk drives (HDDs) are the most popular and costeffective data storage devices nowadays. New technologies are being developed to meet the ever increasing demands for high-capacity and fast data rate HDDs [1]. From the perspective of control engineers, these demands can be translated into the technical specifications that the head position should be accurately maintained along the track center (trackfollowing mode) and swiftly moved from one track to another (track-seeking mode). Traditionally, the HDD head positioner is driven by a voice coil motor (VCM), which however, cannot provide the stringent performance any longer due to its mechanical resonance modes, various disturbances and noises in HDDs. Therefore, the dual-stage actuator (DSA) HDDs are introduced to overcome these limitations [2], [3]. In DSA servo systems, the VCM actuator is used as the primary stage to provide long track seeking but with poor accuracy and slow response time while the secondary stage such as a piezoelectric (PZT) microactuator [4] is used to provide higher precision and faster response but with a stroke limit. By combining the DSA system with properly designed controllers, the overall servo bandwidth of the head positioning mechanism can be significantly increased. Thereby, the DSA HDD system can achieve fast track seeking and allow ultra-high track density, which are far beyond the capability of conventional single-stage HDDs.

The control design for a DSA system is a much greater challenging task than for a conventional single-stage servo with VCM only. This is mainly because a DSA system is a dual-input single-output (DISO) system, which means that

J. Zheng and M. Fu are with the School of Electrical Engineering and Computer Science, The University of Newcastle, Callaghan, NSW 2308, Australia. Jinchuan. Zheng@newcastle.edu.au; Minyue.Fu@newcastle.edu.au for a given desired trajectory, alternative inputs to the two actuators are not unique. Thus, a proper control strategy is required for control allocation in response to external inputs. Otherwise, the two actuators may fight each other and deteriorate the performance instead. A number of approaches have been reported for the DSA control problems. For example, control design for track following and settling can be found in [5]-[7]. In [8], a decoupled track-seeking controller using a three-step design approach is developed to enable highspeed one-track seeking and short-span track-seeking for a dual-stage servo system. Further, short and long-span seeking controls are incorporated in a single control scheme with fast settling time [9], [10].

In most of the previous works, the control designs are focused on individual control problems for either track following or track seeking in HDDs. Accordingly, there needs a mode switching between the controllers for different control tasks. For this purpose, it is typical to employ the initial value compensation method [11] to achieve a smooth switching. This obviously results in extra implementation complexity. To avoid this situation, this paper introduces a unified design method for DSA track-seeking and following control. The control design is based on the doubly coprime factorization (DCF) approach [12], which provides the advantages that: 1) it parameterizes all linear internally stabilized a two-degreeof-freedom (2DOF) controller by two free design parameters; 2 ) it offers a unified design method to solve the seeking and disturbance rejection problems; 3 ) the derived transfer functions of disturbance rejection response and seeking response are simply expressed and they are unique in terms of the design parameters, which makes the relationship between the design parameters and the desired specifications explicit.

Compared with the existing DSA control methods, the proposed 2DOF controller explicitly addresses both track seeking and disturbance rejection problems in a unified design framework and it is easy to implement. In this paper, we focus on the development of the DSA controller for disturbance rejection and step tracking in the PZT range. For track seeking beyond the PZT range, the PZT has little use for reducing the seeking time due to its limited stroke. As such, it is typical to activate the VCM servo only to perform the long seeking task, see [10], [14] for example.

Throughout this paper, we use the following notation. For any signal $u(t)$, we denote its Laplace transform by $\hat{u}(s)$. Let $\mathcal{R} \mathcal{H}_{\infty}$ denote the set of all stable, proper, rational transfer function matrices. 


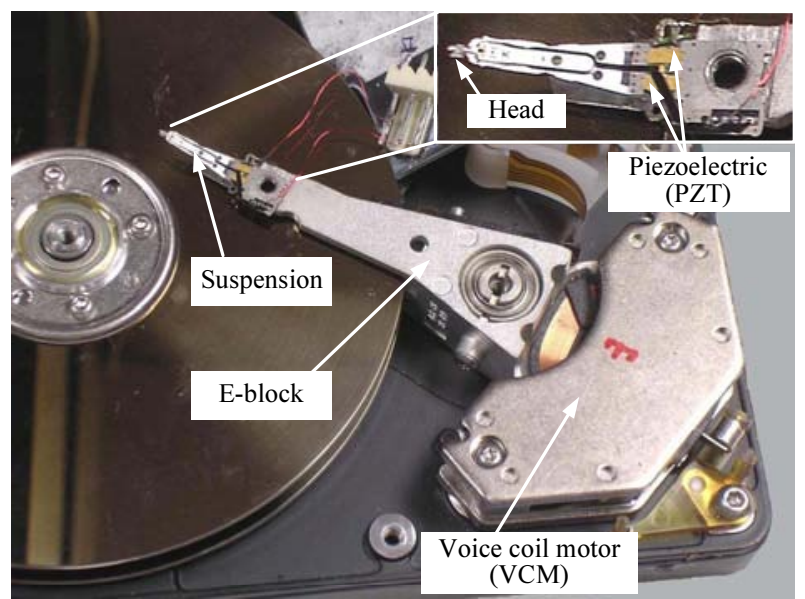

Fig. 1. A HDD DSA system with a PZT microactuator.

\section{Plant and Disturbance Models}

In this paper, we study a dual-stage HDD with a pushpull PZT microactuator as shown in Fig. 1. It consists of a VCM actuator as the primary stage and a PZT actuator as the secondary stage. The PZT is located between the suspension and the E-block, which is moved by the VCM. The two actuators are respectively driven through a PZT amplifier and a VCM driver. The PZT actuator has a stroke limit of \pm 0.5 $\mu \mathrm{m}$. The head position, i.e., our control object, is measured using a laser Doppler vibrometer (LDV) in our experimental setup. The measured frequency responses of the VCM and PZT actuator are shown with the dashed lines in Fig. 2.

The resonances of the E-block and the suspension exerts adverse effects on the tracking performance. Here, we use a compensator with cascaded digital notch filters [10] to actively damp the resonances. The solid lines in Fig. 2 show the measured frequency responses of the VCM and PZT actuator after resonance compensation. From the measured response with resonance compensation, we observe that the compensated VCM actuator and PZT actuator can be reliably approximated as a pure double integrator and a second-order model, respectively in the frequency range of interest. Hence, we approximate the VCM model as

$$
G_{1}=\frac{\hat{y}_{1}}{\hat{u}_{1}}=\frac{k_{1}}{s^{2}}
$$

where $y_{1}$ is the VCM displacement, $u_{1}$ is the VCM control input, and $k_{1}=1.7 \times 10^{8}$. The PZT model is approximated by

$$
G_{2}=\frac{\hat{y}_{2}}{\hat{u}_{2}}=\frac{k_{2}}{s^{2}+a s+b},
$$

where $y_{2}$ is the PZT displacement relative to VCM, $u_{2}$ is the PZT control input, and $k_{2}=4.3 \times 10^{8}, a=3.1 \times 10^{4}$, $b=10^{9}$. Fig. 2 shows that the identified models (dotted lines) can match the measured ones (solid lines) precisely within $3 \mathrm{kHz}$. The system output $y=y_{1}+y_{2}$, i.e., the absolute position of the head position, is the only available measured output for feedback control. Hence, the overall DSA model
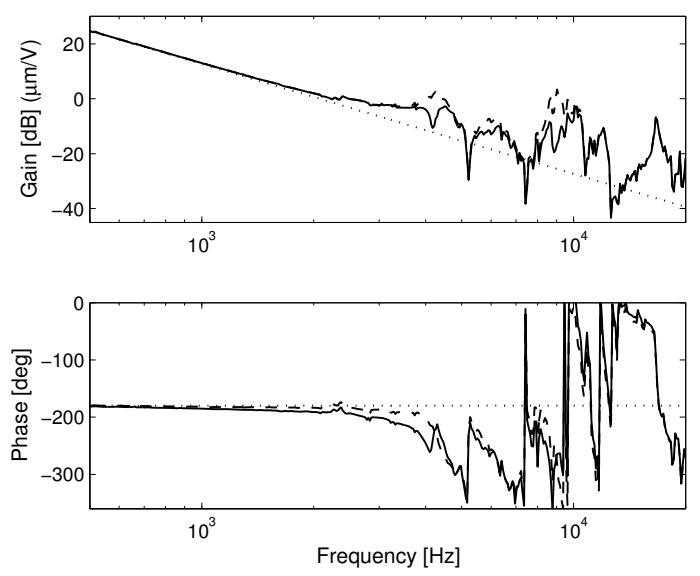

(a) VCM actuator
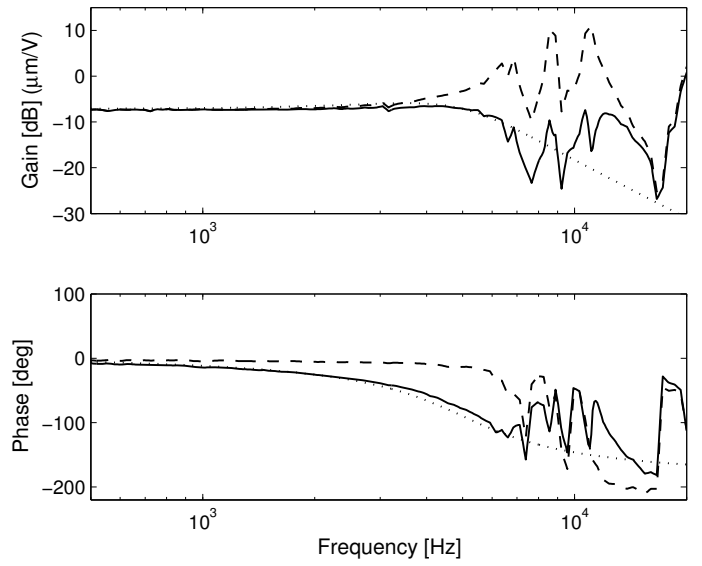

(b) PZT actuator

Fig. 2. Frequency responses of a HDD DSA system (dashed line: measured response; solid line: measured response with resonance compensator; dotted line: simulated response with resonance compensator).

$G$ can be represented as a DISO linear system

$$
\hat{y}=G \hat{u}=\left[\begin{array}{ll}
G_{1} & G_{2}
\end{array}\right]\left[\begin{array}{l}
\hat{u}_{1} \\
\hat{u}_{2}
\end{array}\right] .
$$

From now on we will take the DSA system in (3) as the plant model for control design.

The disturbance sources in HDDs that result in trackfollowing errors contain both repeatable runout (RRO) and nonrepeatable runout (NRRO). In this paper, we will design the controller to reject the disturbances that are reconstructed from a real HDD measurement. Fig. 3 shows the power spectra of the RROs and NRROs extracted from the measured disturbances. We can see that the RRO spectra include the harmonics with a fundamental frequency of $80 \mathrm{~Hz}$ that are associated with the spindle rotation frequency and the written-in RRO while servo writing. On the other hand, the NRROs contain broad-band spectra around $650 \mathrm{~Hz}$ that are caused by the disk flutter and external shock and vibrations. In what follows, the controller will be designed to dedicate to rejecting these RROs and NRROs for reducing the overall position error signal (PES). 


\section{A Unified DSA Control Scheme}

\section{A. Fundamental Design Concept}

Fig. 4 depicts a generic DSA control structure, where $G$ denotes the DSA plant, $K$ the controller to be designed, and the signals $r, y, u$, and $d$ represent, respectively, the seeking command, the head position, the control input, and the disturbances. In this setup, $K$ is essentially a $2 \mathrm{DOF}$ controller with $K_{1}$ the feedforward controller and $K_{2}$ the feedback controller. By using the coprime factorization approach [12], we can parameterize the controller concisely. Let the right and left coprime factorizations of $G$ be given by

$$
G=N D^{-1}=\tilde{D}^{-1} \tilde{N},
$$

where $N, D, \tilde{N}, \tilde{D} \in \mathcal{R H}_{\infty}$ and satisfy the doubly Bezout identify

$$
\left[\begin{array}{cc}
\tilde{X} & -\tilde{Y} \\
-\tilde{N} & \tilde{D}
\end{array}\right]\left[\begin{array}{cc}
D & Y \\
N & X
\end{array}\right]=I
$$

for some $X, Y, \tilde{X}, \tilde{Y} \in \mathcal{R H}_{\infty}$. A DCF representation of the DSA model is given in the Appendix. Hence, the class of all linear internally stabilizing 2DOF controllers $K=\left[\begin{array}{ll}K_{1} & K_{2}\end{array}\right]$ can be parameterized by

$$
\begin{aligned}
\hat{u}= & K_{1} \hat{r}+K_{2} \hat{y} \\
K_{1}= & (\tilde{X}-R \tilde{N})^{-1} Q \\
K_{2}= & (\tilde{X}-R \tilde{N})^{-1}(\tilde{Y}-R \tilde{D}), \\
& Q, R \in \mathcal{R H}_{\infty},
\end{aligned}
$$

where $Q$ and $R$ are the free parameters to be designed. By substituting the controllers $K_{1}, K_{2}$ and the factorized plant model (4) into Fig. 4, we can easily obtain the following input-output relationship in frequency domain

$$
\hat{y}=T_{y r} \hat{r}+S_{y d} \hat{d}
$$

with

$$
\begin{aligned}
T_{y r} & =N Q, \\
S_{y d} & =(X-N R) \tilde{D},
\end{aligned}
$$

where $T_{y r}$ and $S_{y d}$ denote the closed-loop responses from the reference and disturbance to the system output, respectively.

Now, it is obvious that to achieve the optimal track-seeking and following performance is equivalent to selecting a pair of $R$ and $Q$ such that $S_{y d} \equiv 0$ and $T_{y r} \equiv 1$. However, it is more often that only $S_{y d} \rightarrow 0$ and $T_{y r} \rightarrow 1$ is achievable in the frequency range of interest because the plant may not be proper, stable or minimum phase [13]. Therefore, we will next discuss a suboptimal design of $R$ and $Q$ based on the knowledge of the HDD dynamics and the disturbance characteristics.

\section{B. Design of $R$ for Rejecting RROs and NRROs}

The design goal of $R$ is to make the sensitivity function $S_{y d}$ have sufficiently low gains at the frequencies where the RROs and NRROs situate. In addition, $R$ should be capable of allocating the control efforts of the two actuators in response to disturbances at different frequency range. Let
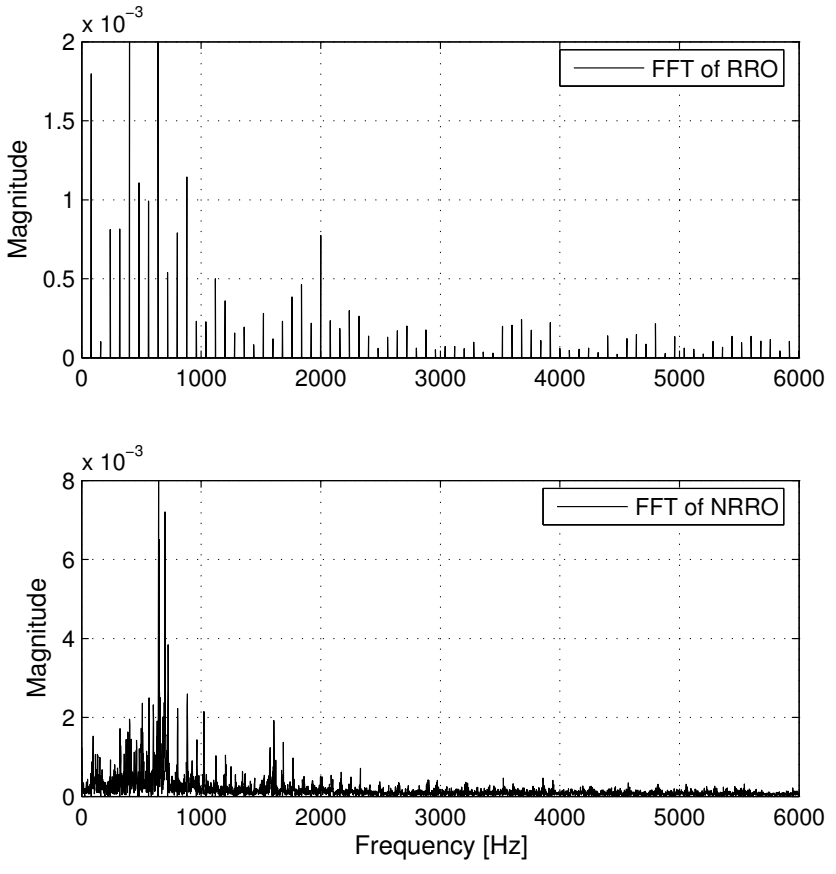

Fig. 3. Power spectra of measured disturbances in a real HDD.

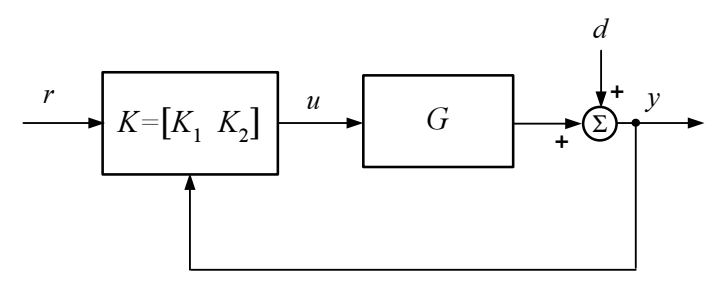

Fig. 4. Generic control structure.

$R=\left[\begin{array}{ll}R_{1} & R_{2}\end{array}\right]^{T}$ and $N=\left[\begin{array}{ll}N_{1} & N_{2}\end{array}\right]$. We also introduce three new stable and proper transfer functions $W, V$ and $\Phi$, and then take

$$
\begin{aligned}
& R_{1}=\frac{X}{N_{1}}(1-W) V \Phi, \\
& R_{2}=\frac{X}{N_{2}}(1-W)(1-V) \Phi,
\end{aligned}
$$

where $W$ depicts desirable gain attenuation of the RROs and NRROs, and $V$ is a low-pass weighting function whose cutoff frequency will determine the control allocation of the two actuators in response to the disturbances. More specific, the VCM is allocated to reject the disturbances below the cut-off frequency, meanwhile the PZT will response to the disturbances above the cut-off frequency due to its highbandwidth characteristics. $\Phi$ is a low-pass filter whose order is chosen to make both $R_{1}$ and $R_{2}$ proper at least. Now, with the expression of $R$ (10)-(11), we have

$$
\begin{aligned}
S_{y d} & =X \tilde{D}(1-\Phi+\Phi W) \\
& \approx X \tilde{D} W . \quad(\text { as } \Phi \approx 1)
\end{aligned}
$$

We can see that the term $X \tilde{D}$ actually represents a nominal sensitivity function (when $R=0$ ) that generally provides basic system robustness in low frequency region. To further 
reject the narrow-band RROs and NRROs at mid- and highfrequency region, $W$ can thus be selected to have low gains at these specified frequencies. One simple choice of $W$ can be given by

$$
W=\prod_{i=1}^{n} \frac{s^{2}+2 \zeta_{1 i} \omega_{i} s+\omega_{i}^{2}}{s^{2}+2 \zeta_{2 i} \omega_{i} s+\omega_{i}^{2}}, \quad \zeta_{1 i}<\zeta_{2 i},
$$

where $n$ is the number of target disturbances for rejection, $\zeta_{1 i}, \zeta_{2 i} \in(0,1)$ are the damping ratios, and $\omega_{i}$ is the center frequencies of RROs and NRROs. Apparently, the gains of $W$ can be arbitrarily low at the disturbance frequencies by selecting appropriate pair $\left(\zeta_{1 i}, \zeta_{2 i}\right)$.

It is also interesting to see from (12) that $S_{y d}$ is unrelated to $V$, which means that various choices of $V$ can return an equivalent $S_{y d}$. This is because the DSA is essentially an actuator-redundant system. However, as the VCM and PZT have quite different mechanical dynamics, it is important to allocate the actuators' responses to the disturbances by choosing a suitable cut-off frequency of $V$. For instance, our experience reveals that for the DSA HDD, $V$ can be chosen as follows

$$
V=\frac{2 \pi f_{c}}{s+2 \pi f_{c}},
$$

where $f_{c}$ denotes the cut-off frequency.

Finally, $\Phi$ is a low-pass filter [15] of the following form

$$
\Phi=\frac{6 \tau^{2} s^{2}+4 \tau s+1}{\tau^{4} s^{4}+4 \tau^{3} s^{3}++6 \tau^{2} s^{2}+4 \tau s+1},
$$

where $\tau$ is the time constant that determines the filter bandwidth. Here, the numerator and denominator order of $\Phi$ are selected such that $\Phi$ has a best fit to unity in both gain and phase characteristics within the bandwidth and additionally $R_{1}$ and $R_{2}$ are made to be proper for practical implementation.

In our case, we choose the design variables of $W$ in (13) to reject the RROs at $80,400,640,880,2000 \mathrm{~Hz}$ and the NRROs around $650 \mathrm{~Hz}$ which are dominant spikes as observed from Fig. 3. Moreover, we set $f_{c}=500 \mathrm{~Hz}$ in (14) and $\tau=1 / 2 \pi 8000$ in (15). Fig. 5 shows the resultant sensitivity function $S_{y d}$ of the DSA system as compared to the single-stage servo with VCM only. We can see that the DSA has a higher 0-dB crossover frequency than that of the VCM and has a lower hump around $1 \mathrm{kHz}$ which is contributed by the PZT loop. Obviously, we can thus expect the DSA servo to have an enhanced disturbance rejection capability than the VCM only. Additionally, the PZT will be dedicated to rejecting the mid- and highfrequency disturbances as specified by $V$.

\section{Design of $Q$ for Track Seeking}

Let $Q=\left[\begin{array}{ll}Q_{1} & Q_{2}\end{array}\right]^{T}$, Due to the fact that $G_{1}$ and $G_{2}$ are minimum phase, we thus aim at the design of $Q_{1}$ and $Q_{2}$ such that $T_{y r}=N_{1} Q_{1}+N_{2} Q_{2} \rightarrow 1$ has a high frequency bandwidth. Furthermore, it is required that the displacement of PZT settles down to zero at steady state. This means that $y_{1}(\infty)=r$ and $y_{2}(\infty)=0$ should be satisfied for a step response with amplitude $r$ assuming the disturbance with

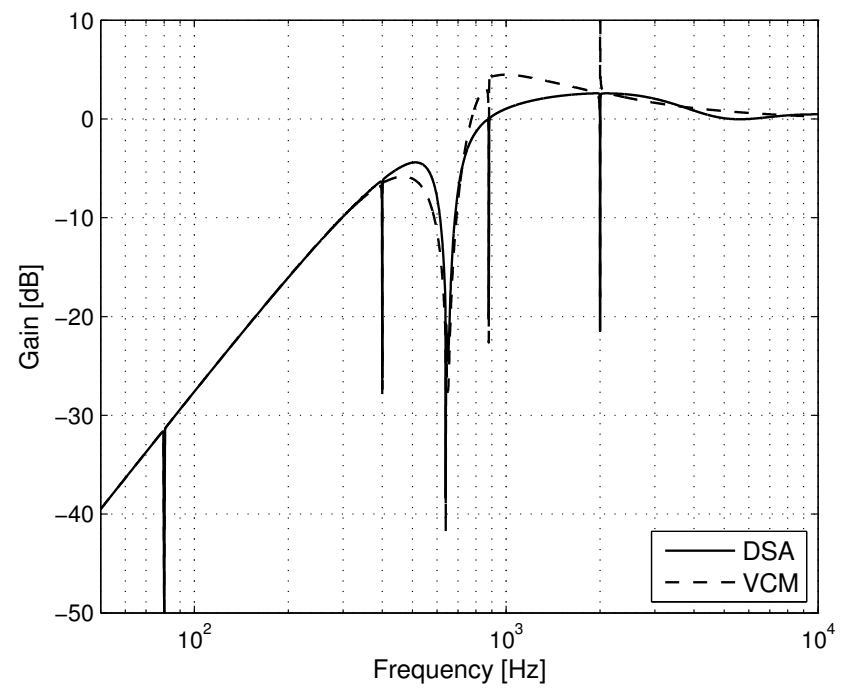

Fig. 5. Comparison of sensitivity functions.

$d(\infty)=0$. Hence, we first analyze the individual position outputs of the two actuators. Partition $D$ as

$$
D=\left[\begin{array}{cc}
D_{1} & 0 \\
0 & D_{2}
\end{array}\right]
$$

and suppose $d=0$, it is thus easy to get

$$
\begin{aligned}
{\left[\begin{array}{l}
\hat{y}_{1} \\
\hat{y}_{2}
\end{array}\right] } & =\left[\begin{array}{cc}
G_{1} & 0 \\
0 & G_{2}
\end{array}\right]\left[\begin{array}{l}
\hat{u}_{1} \\
\hat{u}_{2}
\end{array}\right] \\
& =\left[\begin{array}{cc}
N_{1} D_{1}^{-1} & 0 \\
0 & N_{2} D_{2}^{-1}
\end{array}\right] D Q \hat{r} \\
& =\left[\begin{array}{c}
N_{1} Q_{1} \\
N_{2} Q_{2}
\end{array}\right] \hat{r} .
\end{aligned}
$$

We can see that the step responses of the two actuators are completely decoupled in terms of $Q_{1}$ and $Q_{2}$. As the transfer functions $N_{1}$ and $N_{2}$ are designed (by selecting proper $F$ and $L$ as discussed in the Appendix) to individually reflect the VCM and PZT closed-loop dynamics, we can then interpret $Q_{1}$ and $Q_{2}$ as the trajectory planning functions for the two actuators. We choose $Q_{1}$ and $Q_{2}$ as

$$
\begin{aligned}
& Q_{1}=N_{1}(0)^{-1}, \\
& Q_{2}=\gamma N_{2}(0)^{-1}\left(1-N_{1} N_{1}(0)^{-1}\right),
\end{aligned}
$$

where $\gamma \in\left[\begin{array}{ll}0 & 1\end{array}\right]$ is a tuning scalar. It is obvious that $N_{1}(0) Q_{1}(0)=1$ and $N_{2}(0) Q_{2}(0)=0$, which imply that

$$
y(\infty)=y_{1}(\infty)+y_{2}(\infty)=r+0=r .
$$

Moreover, define the VCM and PZT closed-loop systems by

$$
\begin{aligned}
& T_{1}=N_{1} N_{1}(0)^{-1}, \\
& T_{2}=N_{2} N_{2}(0)^{-1} .
\end{aligned}
$$

We then have the closed-loop transfer function of the DSA

$$
T_{y r}=T_{1}+\gamma T_{2}\left(1-T_{1}\right) .
$$

It is clear that when $\gamma$ varies from 0 to 1 , the cut-off frequency of $T_{y r}$ switches from that of $T_{1}$ to that of $T_{2}$. 


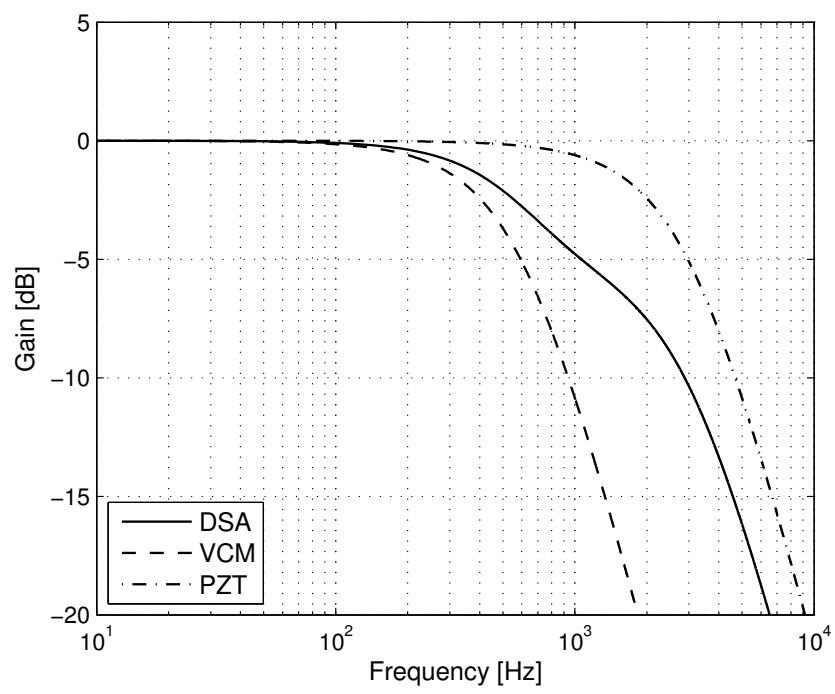

Fig. 6. Frequency response of DSA closed-loop transfer function.

On the other hand, we can see from (19) that the PZT will follow the scaled tracking error of the VCM loop, that is, $\gamma\left(1-N_{1} N_{1}(0)^{-1}\right) r$, where $\gamma$ actually determines the contribution of the PZT to the overall position output. Since the PZT has a faster response than the VCM loop, it is preferable to have a maximal position output of the PZT. Thus, we should maximize $\gamma \in\left[\begin{array}{ll}0 & 1\end{array}\right]$ subject to the condition that the PZT control input $u_{2}$ is within its limit.

In our case, we obtain $\gamma=0.5$ to meet the requirement. Fig. 6 shows the frequency response of the closed-loop systems for the DSA $\left(T_{y r}\right)$, the VCM $\left(T_{1}\right)$, and the PZT $\left(T_{2}\right)$, respectively. We can see that the DSA frequency bandwidth is located between that of the VCM loop and that of the PZT loop, which indicates that the DSA servo system should be faster than the VCM loop but slower than the PZT loop as expected.

\section{Simulation Results}

This section presents simulation results to demonstrate the effectiveness of the proposed DSA controller. These results include the track-following performance for disturbance rejection and track-seeking performance for quick step response.

\section{A. Track-Following Performance}

We first evaluate the PES $(=y)$ of the overall DSA control system in Fig. 4 by setting $r=0$ and injecting the disturbances $d$ characterized by Fig. 3. In this mode, only the feedback controller $K_{2}$ takes action. The power spectra of the resultant PES is shown in Fig. 7. It is obvious that the disturbances at the frequencies as specified in $W$ (13) are remarkably rejected compared to those in Fig. 3. Furthermore, the dual-stage servo significantly decreases the PES amplitude compared with the single-stage servo with VCM only. The PES $\sigma$ value is reduced from 0.0303 to 0.0273 , which is a $10 \%$ reduction ratio. We also see that the dual-stage servo yields a considerable PES reduction

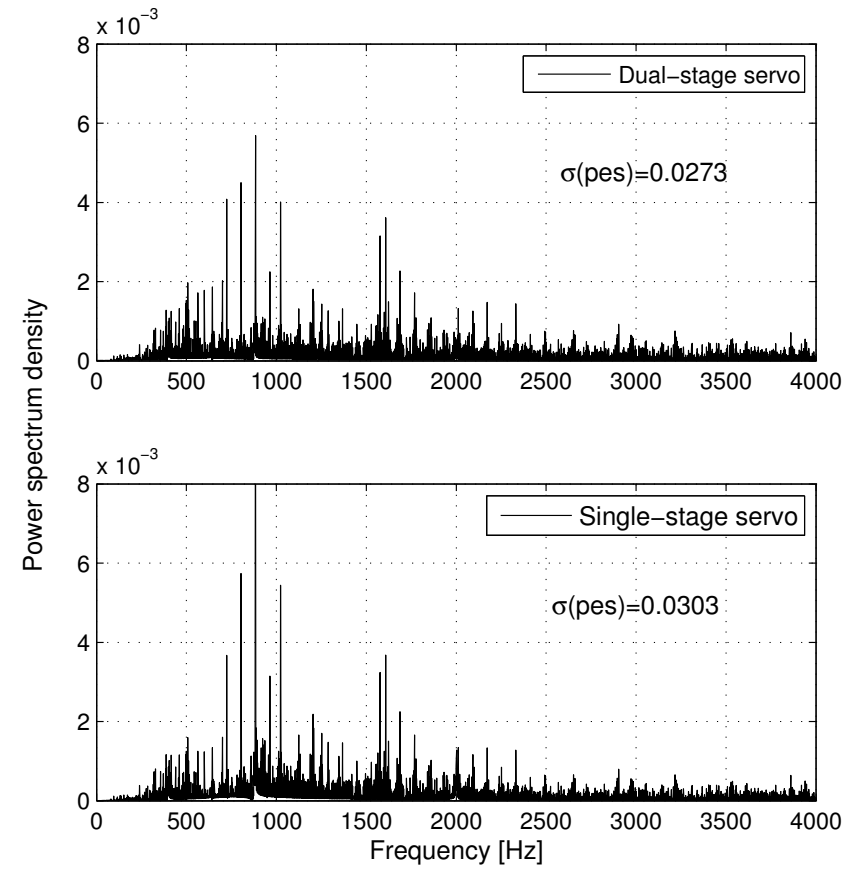

Fig. 7. Power spectra of the PES. The dual-stage servo achieves a $10 \%$ reduction of $\sigma(\mathrm{PES})$ compared with the single-stage servo.

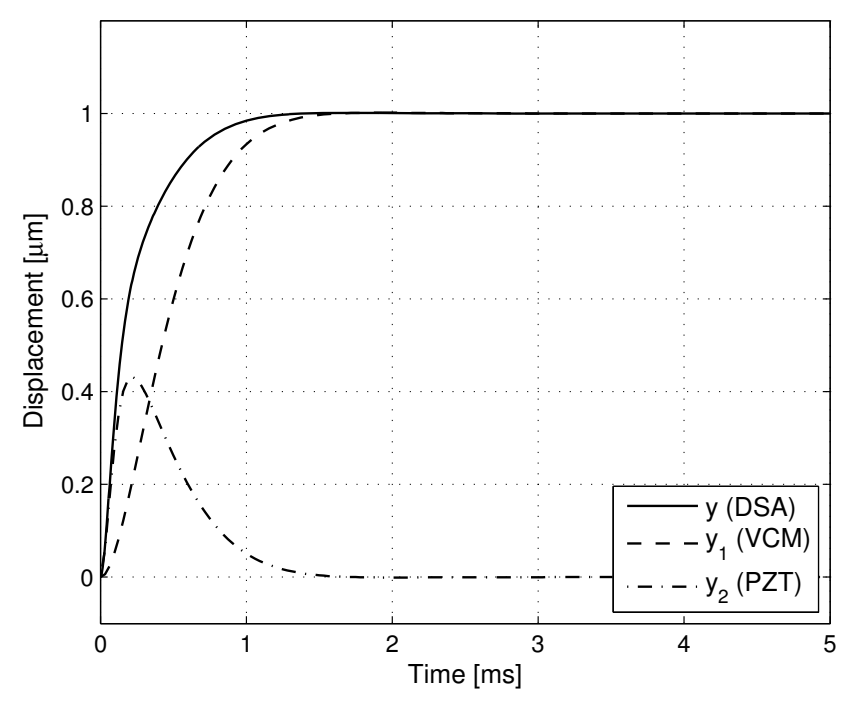

Fig. 8. Track-seeking response.

in the frequency range above $650 \mathrm{~Hz}$ which is the PZT's responsible range as specified by $V$ in (14).

\section{B. Track-Seeking Performance}

The track-seeking performance is evaluated by setting $r=1$ and assuming $d=0$. In this mode, the feedforward controller $K_{1}$ works to generate appropriate seeking trajectories for VCM and PZT, respectively. Fig. 8 shows the track-seeking response. We can see that the settling time under the proposed DSA controller is considerably smaller than that of the VCM. It should be noted that in real implementation, when the head position approaches 
the target, the proposed unified controller does not require extra switching manipulation for transferring to the trackfollowing mode.

\section{CONCLUSION}

In this paper, we have developed a unified approach to track seeking and following control of a HDD DSA system. The design method can explicitly reflect the design criteria of fast seeking and disturbance rejection in terms of two design parameters. We have further discussed the selection of the design parameters for proper DSA control allocation. Finally, simulation results are presented to verify the efficacy of the proposed controller. It can be concluded that the proposed unified controller can achieve desirable performances for both track-seeking and following that are equivalent to that by two separate conventional controllers. Therefore, the developed HDD DSA controller is comparatively simpler for implementation.

\section{APPENDIX}

\section{A FACTORIZATION REPRESENTATION OF DSA MODEL}

First, let the DSA model $G(s)$ in (3) be represented in state-space as follows

$$
G(s)=C(s I-A)^{-1} B,
$$

with

$$
\begin{aligned}
& A=\left[\begin{array}{c|c}
A_{1} & 0 \\
\hline 0 & A_{2}
\end{array}\right]=\left[\begin{array}{cc|cc}
0 & 1 & 0 & 0 \\
0 & 0 & 0 & 0 \\
\hline 0 & 0 & 0 & 1 \\
0 & 0 & -b & -a
\end{array}\right], \\
& B=\left[\begin{array}{c|c}
0 & 0 \\
B_{1} & 0 \\
\hline 0 & B_{2}
\end{array}\right]=\left[\begin{array}{c|c}
k_{1} & 0 \\
\hline 0 & 1 \\
0 & k_{2}
\end{array}\right], \\
& C=\left[\begin{array}{ll|ll}
1 & 0 & 1 & 0
\end{array}\right],
\end{aligned}
$$

where $A_{1,2}, B_{1,2}$ and $C_{1,2}$ are the state-space representation of the VCM and PZT, respectively. Since the pairs $(A, B)$ and $(A, C)$ are stabilizable and detectable, respectively. we can select $F$ and $L$ such that $(A-B F)$ and $(A-L C)$ are both Hurwitz. Thus, a DCF of $G(s)$ [16] is given by

$$
\left\{\begin{array}{l}
N(s)=C(s I-A+B F)^{-1} B \\
D(s)=I-F(s I-A+B F)^{-1} B \\
\tilde{N}(s)=C(s I-A+L C)^{-1} B \\
\tilde{D}(s)=I-C(s I-A+L C)^{-1} L \\
X(s)=I+C(s I-A+B F)^{-1} L \\
\tilde{X}(s)=I+F(s I-A+L C)^{-1} B \\
Y(s)=-F(s I-A+B F)^{-1} L \\
\tilde{Y}(s)=-F(s I-A+L C)^{-1} L .
\end{array}\right.
$$

It can be seen that the DCF representation of the DSA model is expressed by explicit formulas in terms of its state-space realization. Hence, it is numerically easy to use.

Now, to obtain (25) is reduced to choose two gains $F$ and $L$ which actually represent the state feedback gain matrix and state estimator gain matrix of the DSA model, respectively. Since the coupling between the VCM and the PZT is negligible, the gains $F$ and $L$ can be partitioned as

$$
F=\left[\begin{array}{c|c}
F_{1} & 0 \\
\hline 0 & F_{2}
\end{array}\right], \quad L=\left[\begin{array}{c}
L_{1} \\
\hline L_{2}
\end{array}\right] .
$$

Hence, we can individually design the gains for the VCM and PZT loops by using the pole placement method [14] such that the PZT loop should have a faster dynamics than the VCM loop, and the estimator is faster than the state feedback loop. To do this, we select $F_{1}=\left[0.08364 \times 10^{-5}\right]$ and $L_{1}=\left[\begin{array}{ll}1.0 \times 10^{5} & 1.4 \times 10^{9}\end{array}\right]^{T}$ to make the VCM loop and its estimator have a bandwidth of 600 and $3000 \mathrm{~Hz}$, respectively, and select $F_{2}=-\left[\begin{array}{ll}1.5 & 6.8 \times 10^{-6}\end{array}\right]$ and $L_{2}=$ $\left[-104519.4 \times 10^{8}\right]^{T}$ for the counterparts of the PZT with 5 and $6 \mathrm{kHz}$ bandwidths, respectively. Then, the DCF of $G$ can be easily computed by (25).

\section{REFERENCES}

[1] R. Wood and H. Takano, "Prospects for magnetic recording over the next 10 years," in Proc. IEEE Int. Magnetics Conference, 2006, pp. 98.

[2] K. Mori, T. Munemoto, H. Otsuki, Y. Yamaguchi, and K. Akagi, "A dual-stage magnetic disk drive actuator using a piezoelectric device for a high track density," IEEE Trans. Magn., vol. 27, no. 6, pp. 52985300, Nov. 1991.

[3] T. Imamura, M. Katayama, Y. Ikegawa, T. Ohwe, R. Koishi, and T. Koshikawa, "MEMS-based integrated head/actuator/slider for hard disk drives," IEEE/ASME Trans. Mechatron., vol. 3, no. 3, pp. 166174, Sep. 1998

[4] R. Evans, J. Griesbach, and W. Messner, "Piezoelectric microactuator for dual stage control,” IEEE Trans. Magn., vol. 35, no. 2, pp. 977-982, Mar. 1999.

[5] S. Schroeck, W. Messner, and R. McNab, "On compensator design for linear time-invariant dual-input single-output systems," IEEE/ASME Trans. Mechatron., vol. 6, no. 1, pp. 50-57, Mar. 2001.

[6] X. Huang, and R. Horowitz, "Robust controller design of a dual-stage disk drive servo system with an instrumented suspension," IEEE Trans. Magn., vol. 41, no. 8, pp. 2406-2413, Aug. 2005.

[7] H. Numasato and M. Tomizuka, "Settling control and performance of a dual-actuator system for hard disk drives," IEEE/ASME Trans. Mechatron., vol. 8, no. 4, pp. 431-438, Dec. 2003.

[8] M. Kobayashi, and R. Horowitz, "Track seek control for hard disk dual-stage servo systems," IEEE Trans. Magn., vol. 37, no. 2, pp. 949-954, Mar. 2001.

[9] B. Hredzak, G. Herrmann, and G. Guo, "A proximate-time-optimal control design and its application to a hard disk drive dual-stage actuator system," IEEE Trans. Magn., vol. 42, no. 6, pp. 1708-1715, Jun. 2006.

[10] J. Zheng, M. Fu, Y. Wang, and C. Du, "Nonlinear tracking control for a hard disk drive dual-stage actuator system," IEEE/ASME Trans. Mechatron., vol. 13, no. 5, pp. 510-518, Oct. 2008.

[11] T. Yamaguchi, H. Numasato, and H. Hirai, "A mode-switching control for motion control and its application to disk drives: design of optimal mode-switching conditions," IEEE/ASME Trans. Mechatron., vol. 3, no. 3, pp. 202-209, Sep. 1998.

[12] M. Vidyasagar, Control System Synthesis: A Factorization Approach, Cambridge, MA: MIT Press, 1995.

[13] J. Zheng, W. Su, and M. Fu, "Tow-degree-of-freedom of control of a dual-stage actuator positioning system for shor-span tracking," in Proc. American Control Conf., 2009, pp. 3681-3686.

[14] G. F. Franklin, J. D. Powell, and A. Emami-Naeini, Feedback Contro of Dynamic Systems, 3rd ed., Reading, MA: Addison-Wesley, 1994.

[15] Y. Choi, K. Yang, W. Chung, H. Kim, and I. Suh, "On the robustness and performance of disturbance observers for second-order systems," IEEE Trans. Automat. Contr., vol. 48, no. 2, pp. 315-320, Feb. 2003.

[16] C. Nett, C. Jacobson, and M. Balas, "A connection between state-space and doubly coprime fractional representations," IEEE Trans. Automat. Contr., vol. AC-29, no. 9, pp. 831-832, Sep. 1984. 